http://jmscr.igmpublication.org/home/

ISSN (e)-2347-176x ISSN (p) 2455-0450

crossref DOI: https://dx.doi.org/10.18535/jmscr/v7i7.160

Journal Of Medical Science And Clinical Research

\title{
Laboratory Risk Indicator in Necrotizing Fasciitis (LRINEC) Score- An Early Diagnostic Tool for Necrotizing Fasciitis
}

\author{
Author \\ Dr Snigdh Garg \\ Academic Junior Resident (2017-20), Department of General Surgery, Darbhanga Medical College \& \\ Hospital, Darbhanga, Bihar, India
}

\begin{abstract}
Objective: To assess the utility of Laboratory Risk Indicator in Necrotizing Fasciitis (LRINEC) Score in cases of Necrotizing Fasciitis as an early diagnostic tool. Necrotizing Fasciitis is a rapidly progressive inflammation of the fascia with secondary necrosis of the subcutaneous tissues. It requires aggressive surgical intervention to combat associated high morbidity and mortality.

Methods: A retrospective analysis of cases admitted in Darbhanga Medical College and Hospital from Sep 2017 to Feb 2019 having diagnosis of Necrotizing Fasciitis at time of admission was done. LRINEC Scoring was done for each of them and confirmation of diagnosis was done by biopsy of tissue samples from the patients. Sensitivity, Specificity, PPV, NPV and Likelihood Ratios were computed for patients taking LRINEC Scores of $\geq 6$ as a cutoff for diagnosis.

Results: Thirty five patients admitted with an initial diagnosis of Necrotizing Fasciitis were eligible for the study. Out of these, fourteen patients had biopsy-proven Necrotizing Fasciitis. Taking LRINEC Score $\geq 6$ as a cutoff, Sensitivity of $85.7 \%$, Specificity of $67 \%$, PPV of $63.2 \%$ and NPV of $87.5 \%$ for diagnosing Necrotizing Fasciitis was obtained. The Likelihood ratios for a positive and negative biopsy were 2.6 and 0.2 respectively.

Conclusion: LRINEC Score of $\geq 6$ can be used as a cutoff for diagnosis of Necrotizing Fasciitis along with clinical suspicion and using tissue biopsy as a confirmatory test. It should not be used in isolation, as that will lead to a high number of false positives.
\end{abstract}

Keywords: LRINEC Score, Necrotizing Fasciitis.

\section{Introduction}

Necrotizing Fasciitis is a rapidly progressive inflammation of the fascia with secondary necrosis of the subcutaneous tissues. It spreads from subcutaneous tissue along superficial and deep fascial planes facilitated by bacterial enzymes and toxins. Infection causes vascular occlusion, ischemia and tissue necrosis along with damage of superficial nerves leading to characteristic localized anaesthesia. Muscles are usually spared. $80 \%$ patients have history of previous trauma or infection. $60 \%$ cases commence in lower extremities. Fournier's Gangrene is a form of necrotizing fasciitis localized to scrotum and perineal areas. Meleney's synergistic gangrene involves lower abdomen and thorax, usually in immunocompromised patients post surgery. Necrotizing Fasciitis can be caused by aerobic, anaerobic or a mixed flora. It is classified as: 
Type I (80\%) - polymicrobial- Gram positive cocci (Staphylococcus, non Grp A Streptococci, enterococci), Gram negative bacilli(Escherichia coli, Pseudomonas, Proteus) and Anaerobes (Bacteroides and Clostridial species). Clostridial species (C.perfringens, C.septicum and C.sordellii) account for $10 \%$ of Type 1 infection; common in perineum, trunk and post operative wounds; common in Diabetics and immunocompromised; usually not associated with trauma.

Type II- monomicrobial- Grp A Streptococci or MRSA; $2^{\text {nd }}$ most common type; common in extremities; common in young individuals and those without comorbidities; associated with history of injury.

Type III- due to Vibrio vulnificus (Gram negative bacillus), a bacterium found in saltwater; rare type; associated with chronic liver disease, DM, steroid therapy, CKD; occurs after minor trauma.

Type IV- due to fungal infection, mostly Aspergillus zygomycetes; also a rare type. Mean age group involved is 38-44 yrs with a male: female ratio of 2-3:1. These infections are rapidly progressive and can be difficult to differentiate from soft tissue infections. It requires aggressive surgical intervention to combat associated high morbidity and mortality.

Risk factors: Diabetes mellitus, smoking, old age, penetrating trauma, pressure sores, immunosuppression (including steroid therapy, HIV infection), alcoholism, malnourishment, IV drug abuse, perineal infection(perineal abscess, Bartholin's cyst), skin infection or trauma(boils, bites, abrasions), obesity, peripheral arterial disease.

Clinical Signs: edema stretching beyond skin erythema, woody hard texture of subcutaneous tissue, inability to distinguish fascial planes and muscle groups on palpation, disproportionate pain in relation to affected area, skin vesicles, soft tissue crepitus, absent lymphangitis, elevated temperature, tachycardia, rapid progression to septic shock, SIRS,MODS. Finger test is positive. Clinical stages:

1) fever, erythema, edema, tenderness

2) blisters, bullae formation

3) hypoaesthesia or anaesthesia, crepitus, hemorrhagic bullae

Necrotizing Fasciitis is a surgical emergency. Diseased area should be debrided without delay until viable healthy bleeding tissue is reached. Mortality is around $30-50 \%$.

LRINEC (Laboratory Risk Indicator in Necrotizing Fasciitis) Score considers the following variables- CRP ( $<150$ or $\geq 150 \mathrm{mg} / \mathrm{L}$ ), TLC $(<15,15-25$ or $>25$ per cubic $\mathrm{mm}), \mathrm{Hb}(<11$, $11-13.5$ or $>13.5 \mathrm{~g} / \mathrm{dL})$, Serum Sodium $(<135$ or $\geq 135 \mathrm{mmol} / \mathrm{L})$, Serum Creatinine $(\leq 1.6$ or $>1.6$ $\mathrm{mg} / \mathrm{dL})$ and Blood Glucose $(\leq 180$ or $>180 \mathrm{mg} / \mathrm{dL})$ with highest weight age given to CRP levels. Minimum score is 0 and maximum score is 13 . Table 1 illustrates the method of calculation of LRINEC Score.

Table 1 LRINEC (Laboratory Risk Indicator in Necrotizing Fasciitis) Score

\begin{tabular}{|l|l|c|c|}
\hline S.No. & Variables & Value & Score \\
\hline 1. & C Reactive Protein (CRP) & $\geq 150 \mathrm{mg} / \mathrm{L}$ & 0 \\
\hline & & $\geq 150 \mathrm{mg} / \mathrm{L}$ & 4 \\
\hline 2. & Total Leucocyte Count (TLC) & $<15 \times 10^{3}$ per cubic $\mathrm{mm}$ & 0 \\
\hline & & $15-25 \times 10^{3} \mathrm{per}$ cubic $\mathrm{mm}$ & 1 \\
\hline & & $>25 \times 10^{3} \mathrm{per} \mathrm{cubic} \mathrm{mm}$ & 2 \\
\hline 3. & Haemoglobin $(\mathrm{Hb})$ & $>13.5 \mathrm{~g} / \mathrm{dL}$ & 0 \\
\hline & & $11-13.5 \mathrm{~g} / \mathrm{dL}$ & 1 \\
\hline & & $<11 \mathrm{~g} / \mathrm{dL}$ & 2 \\
\hline 4. & Serum Sodium & $<135 \mathrm{mmol} / \mathrm{L}$ & 0 \\
\hline & & $\leq 135 \mathrm{mmol} / \mathrm{L}$ & 2 \\
\hline 5. & Serum Creatinine & $>1.6 \mathrm{mg} / \mathrm{dL}$ & 0 \\
\hline & & $\leq 180 \mathrm{mg} / \mathrm{dL}$ & 2 \\
\hline 6. & Blood Glucose & $>180 \mathrm{mg} / \mathrm{dL}$ & 0 \\
\hline & & & 1 \\
\hline
\end{tabular}




\section{Methods}

A retrospective study was undertaken of patients admitted to Darbhanga Medical College and Hospital, a tertiary care hospital in North Bihar region of India, having a diagnosis of Necrotizing Fasciitis upon admission during the period of Sep 2017 to Feb 2019. The patients whose LRINEC Score was obtained within $24 \mathrm{hrs}$ from admission were considered for the study. Tissue biopsy report of patients was taken as the gold standard investigation to establish the diagnosis of Necrotizing fasciitis. The patients were divided into two groups- one, with biopsy proven Necrotizing Fasciitis and the other, in which biopsy was negative for Necrotizing Fasciitis. LRINEC Score of $\geq 6$ was considered as cutoff for diagnosis of Necrotizing Fasciitis and this was compared with result of tissue biopsy report to obtain positive and negative predictive values of LRINEC Score as a diagnostic test. A $2 \times 2$ table was made for analysis and Sensitivity, Specificity, Positive Predictive Value (PPV) and Negative Predictive Value (NPV) were obtained. Likelihood ratios for positive and negative tissue biopsy were also calculated.

\section{Results}

There were a total of 35 patients admitted with a diagnosis of Necrotizing Fasciitis in a tertiary care hospital in North Bihar, in the period of Sep 2017 to Feb 2019, whose LRINEC Score was obtained within $24 \mathrm{hrs}$ from admission. Out of these 35 patients, 14 patients had a positive tissue biopsy for Necrotizing Fasciitis and remaining 21 patients had a negative tissue biopsy for Necrotizing Fasciitis. 3 patients amongst those with biopsy proven Necrotizing Fasciitis died, so the mortality in this study was $21 \%$. Table 2 provides details of all patients with positive tissue biopsy for Necrotizing Fasciitis and Table 3 shows details of patients with negative tissue biopsy for Necrotizing Fasciitis.
Table 2 LRINEC Scores of patients with Positive Tissue Biopsy for Necrotizing Fasciitis

\begin{tabular}{|l|c|c|c|}
\hline Patient & Age & Gender & LRINEC Score \\
\hline 1 & 57 & M & 8 \\
\hline 2 & 46 & M & 9 \\
\hline 3 & 60 & F & 6 \\
\hline 4 & 9 & M & 5 \\
\hline 5 & 29 & F & 6 \\
\hline 6 & 44 & M & 6 \\
\hline 7 & 50 & M & 11 \\
\hline 8 & 64 & M & 7 \\
\hline 9 & 31 & M & 8 \\
\hline 10 & 35 & M & 7 \\
\hline 11 & 26 & M & 5 \\
\hline 12 & 42 & F & 8 \\
\hline 13 & 61 & M & 10 \\
\hline 14 & 52 & M & 8 \\
\hline
\end{tabular}

Table 3 RINEC Scores of patients with Negative Tissue Biopsy for Necrotizing Fasciitis

\begin{tabular}{|l|c|c|c|}
\hline Patient & Age & Gender & LRINEC Score \\
\hline 15 & 40 & M & 1 \\
\hline 16 & 24 & M & 0 \\
\hline 17 & 60 & M & 5 \\
\hline 18 & 35 & M & 5 \\
\hline 19 & 41 & F & 7 \\
\hline 20 & 29 & F & 0 \\
\hline 21 & 26 & M & 2 \\
\hline 22 & 11 & M & 6 \\
\hline 23 & 44 & M & 3 \\
\hline 24 & 59 & M & 1 \\
\hline 25 & 37 & M & 6 \\
\hline 26 & 56 & M & 3 \\
\hline 27 & 77 & M & 7 \\
\hline 28 & 8 & F & 0 \\
\hline 29 & 33 & M & 8 \\
\hline 30 & 43 & M & 2 \\
\hline 31 & 25 & M & 6 \\
\hline 32 & 44 & F & 3 \\
\hline 33 & 68 & M & 5 \\
\hline 34 & 51 & M & 1 \\
\hline 35 & 49 & M & 7 \\
\hline & & & \\
\hline
\end{tabular}

Considering the generally accepted LRINEC Score of $\geq 6$ as cutoff for Necrotizing Fasciitis diagnosis, sensitivity of $85.7 \%$, specificity of $67 \%$, PPV of $63.2 \%$ and NPV of $87.5 \%$ was obtained. Lowering the cutoff value of LRINEC Score to $\geq 5$, lead to sensitivity of $100 \%$, specificity of $52.4 \%$, PPV of $58.33 \%$ and NPV of $100 \%$. Taking up LRINEC Score of $\geq 8$ as cutoff, sensitivity of $50 \%$, specificity of $95 \%$, PPV of $87.5 \%$ and NPV of $74 \%$ was obtained. 
Likelihood Ratios (LR) were also calculated for obtaining a positive or negative tissue biopsy in patients having LRINEC cutoff score $\geq 6, \geq 5$ and $\geq 8$. LR are used for assessing the value of performing a diagnostic test. They determine whether a test result usefully changes the probability that a condition exists. $\mathrm{LR}+$ indicates LR for positive result and LR- indicates LR for negative result. A LR result of $<0.2$ or $>5$ would have a moderate to large effect on the post test probability. For LRINEC Score of $\geq 6$, LR+ was 2.57 and LR- was 0.21. For LRINEC Score of $\geq 5$, LR+ was 2.1 and LR- was 0. For LRINEC Score of $\geq 8, L R+$ was 10 and LR- was 0.53. This indicates that patients with LRINEC Score of $<5$ have a high chance of having a negative tissue biopsy for Necrotizing Fasciitis and those with a score of $\geq 8$ have a high chance of having a positive tissue biopsy for Necrotizing Fasciitis.

\section{Discussion}

From this study it is evident that taking LRINEC Score cutoff of $\geq 6$ provides a sensitivity of $85.7 \%$, specificity of $67 \%$, PPV of $63.2 \%$ and NPV of $87.5 \%$ with LR+ of 2.57 and LR- of 0.21 . Hence a LRINEC Score of $\geq 6$ cannot be considered as a confirmatory diagnosis of Necrotizing Fasciitis but, with further investigations like tissue biopsy and clinical suspicion, can be used to hasten the diagnosis of Necrotizing Fasciitis. Considering the fact that Necrotizing Fasciitis is a serious medical condition with rapid progression of disease, LRINEC Score serves the purpose of providing early diagnosis and therefore reduces the mortality and morbidity form it by allowing early surgical intervention in needed cases. Further, in patients having a LRINEC Score of $\geq 8$, this study shows a specificity of $95 \%$ and LR+ of 10 . Hence, in such patients LRINEC Score can be a reliable indicator of diagnosis of Necrotizing Fasciitis and there would be very high chances of obtaining a positive tissue biopsy. Since these patients are likely to have a severe disease, hence early surgical intervention in them would be highly beneficial. In patients with LRINEC Score of $<5$, the chances of diagnosis of Necrotizing Fasciitis are very less. This study shows that for a LRINEC Score cutoff of $\geq 5$, sensitivity is $100 \%$, NPV is $100 \%$ and LR- is 0 , implying that there is a very high negative tissue biopsy rate for Necrotizing Fasciitis in patients with LRINEC Score of <5. In such patients a conservative approach could be considered and early surgical intervention should be withheld until confirmatory diagnosis of Necrotizing Fasciitis has been established as these patients are more likely to have soft tissue infections.

Wong et al proposed LRINEC score in 2004 by doing a retrospective observational study of 145 patients with Necrotizing Fasciitis and 309 patients with severe abscess or cellulitis. They obtained a PPV of $92 \%$ and NPV of $96 \%$ with a LRINEC Score cutoff of $\geq 6$. This led them to conclude that LRINEC Score was capable of detecting even early cases of Necrotizing Fasciitis and patients with a score of $\geq 6$ should be carefully evaluated for presence of Necrotizing Fasciitis. $^{[1],[2]}$

MJ Holland, in 2009, presented a retrospective analysis of 28 patients having admission diagnosis of Necrotizing Fasciitis to assess applicability and usefulness of LRINEC Score. He concluded that LRINEC Score cutoff of $\geq 6$ by itself, is not sufficient to rule in or out the diagnosis of Necrotizing Fasciitis. $\left[{ }^{3]}\right.$ Burner et al, in 2016, also concluded in their study that LRINEC Score used in isolation is not sufficiently sensitive to rule out Necrotizing Fasciitis in population presenting to emergency department. ${ }^{[4]}$

Impact of LRINEC Score in decreasing mortality due to early diagnosis of Necrotizing Fasciitis was elucidated by Liao et al and Mukhopadhyay in their studies conducted in 2012 and 2016 respectively. ${ }^{[5],[6]}$ Bechar et al, in 2017, stated in their study that LRINEC Score is a useful clinical determinant in diagnosis and surgical treatment of patients with Necrotizing Fasciitis with a statistically positive correlation between LRINEC Score and true diagnosis of Necrotizing Fasciitis. ${ }^{[7]}$ Narasimhan et al stated in their study, 
conducted in 2018, that LRINEC Score can be used as an adjunct to early diagnosis of Necrotizing Fasciitis though a high degree of clinical suspicion remains the most important factor in its early diagnosis. ${ }^{[8]}$

\section{Conclusion}

LRINEC Score is based upon the biochemical changes associated with septicemia which occurs in cases of Necrotizing Fasciitis. Quantifying these changes and utilizing them for scoring purpose to facilitate diagnosis of Necrotizing Fasciitis is a pragmatic approach. The point of contention lies in setting an optimum cutoff of LRINEC Score. Taking a LRINEC Score cutoff of $\geq 5$ would lead to inclusion of almost all cases but with a very high negative tissue biopsy rate as well as inadvertent surgical interventions, which are undesirable. On the contrary, a LRINEC Score cutoff of $\geq 8$ would lead to a high positive tissue biopsy rate along with improved outcome with early surgical intervention but at the risk of missing out some cases of Necrotizing Fasciitis, which may lead to a potentially higher morbidity and mortality. In light of the data obtained, it can be stated that LRINEC Score of $\geq 6$ can be used as a cutoff for diagnosis of Necrotizing Fasciitis along with clinical suspicion and a more aggressive diagnostic regimen, using tissue biopsy as a confirmatory test. It should not be used in isolation, as that will lead to a high number of false positives.

\section{References}

1. Wong CH, Khin LW, Heng KS, Tan KC, Low CO. The LRINEC (Laboratory Risk Indicator for Necrotizing Fasciitis) score: a tool for distinguishing necrotizing fasciitis from other soft tissue infections. Critical Care Medicine 2004; 32:1535-1541.

2. Wong $\mathrm{CH}$, Khin LW. Clinical relevance of LRINEC Score for assessment of early Necrotizing Fasciitis. Critical Care Medicine 2005; 33(7):1677.
3. Holland MJ. Application of the Laboratory Risk Indicator in Necrotizing Fasciitis (LRINEC) score to patients in a tropical tertiary referral centre. Anaesthesia and Intensive Care 2009; 37(4):588-592.

4. Burner E, Henderson SO, Burke G, Nakashioya J, Hoffman JR. Inadequate sensitivity of laboratory risk indicator to rule out necrotizing fasciitis in the emergency department. Western Journal of Emergency Medicine 2016; 17(3):336.

5. Liao CI, Lee $\mathrm{YK}, \mathrm{Su} \mathrm{YC}$, Chuang $\mathrm{CH}$, Wong $\mathrm{CH}$. Validation of the LRINEC Score for early diagnosis of Necrotizing Fasciitis. Tzu Chi Medical Journal 2012; 24(2):73-76.

6. Mukhopadhyay M. Necrotizing soft tissue infections: The role of the LRINEC score. Hellenic Journal of Surgery 2016; 88(1):31-34.

7. Bechar J, Sepehripour S, Hardwicke J, Filobbos G. LRINEC Score foe assessment of early Necrotizing Fasciitis: a systematic review of the literature. The Annals of The Royal College of Surgeons of England 2017; 99(5):341-346.

8. Narasimhan V, Ooi G, Weidlich S, Carson P. LRINEC Score for early diagnosis of Necrotizing Fasciitis in Darwin. ANZ Journal of Surgery 2018; 88(1-2):E45-E49. 